\title{
Erupting Compound Odontome - A case report
}

\author{
Dr. Sahana Srinath ${ }^{1}$, Dr. Kendole Rohit ${ }^{2}$, Dr. Srinath S.K. ${ }^{3}$ \\ ${ }^{I}$ (Professor and Head, Department of oral and maxillofacial pathology, Government Dental College and \\ Research Institute, Bangalore, India) \\ ${ }_{2}^{2}$ (Post graduate student, Department of oral and maxillofacial pathology, Government Dental College and \\ Research Institute, Bangalore, India) \\ ${ }_{3}^{3}$ (Professor, Department of Pedodontics and Preventive dentistry, Government Dental College and Research \\ Institute, Bangalore, India)
}

\begin{abstract}
Odontomas are hamartomatous lesions composed of various dental tissues, i.e., enamel, dentin, cementum and sometimes pulp. They are the most common benign odontogenic tumours, constituting $22 \%$ of all odontogenic tumours of the jaw. They are slow-growing, benign tumours showing nonaggressive behaviour. They are usually asymptomatic and are often discovered during routine radiography. We report a case of erupting compound odontome which clinically simulated the erupting permanent incisors in an 8 year old boy, which was associated with an unerupted maxillary left central and lateral permanent incisors. The diagnosis was confirmed after surgical removal and histopathological analysis.
\end{abstract}

Key Words: complex odontoma, compound odontoma, denticles, odontoma,

\section{Introduction:}

The term odontoma refers to any tumour of odontogenic origin. An odontoma is a growth in which both epithelial and mesenchymal cells exhibit complete differentiation resulting in functional ameloblasts and odontoblasts which form enamel and dentin respectively. This enamel and dentin are usually laid down in an abnormal pattern due to failure of organization of the odontogenic cells to reach a normal state of morphodifferentiation ${ }^{1}$

Paul Broca was the first to coin the term "odontoma" in $1867^{2}$. The etiology of odontomas has been attributed to various pathological conditions like local trauma, inflammatory and/or infectious process, hereditary anomalies (Gardners syndrome, Hermanns syndrome), odontoblastic hyperactivity, and alterations in the genetic component responsible for controlling dental development. The persistence of a portion of dental lamina may be an important factor in the etiology of a compound or complex odontoma and either of this may occur instead of a tooth ${ }^{3}$. It has also been proposed that odontomas are inherited from a mutant gene or possible postnatal interference with the genetic control of tooth development. Odontomas are often non-aggressive and slow growing in nature ${ }^{4}$.

According to the latest classification of the World Health Organization (WHO, 2005), two types of odontomas can be found: complex odontomas and compound odontomas - the latter being twice as common as the former ${ }^{2}$.

Compound odontomas are usually seen in the anterior maxilla, over the crowns of unerupted teeth, or between the roots of erupted teeth. Radiographically, these lesions are usually unilocular and contain multiple radio-opaque, miniature tooth-like structures known as denticles ${ }^{5}$.

Complex odontomas are found in the posterior mandibular region, over impacted teeth, and can reach several centimeters in size. Radiographically, these lesions show a radio-opaque mass with occasional nodular elements, which is surrounded by a fine radiolucent zone. The lesions are separated from the normal bone by a well-defined corticalization line. Radiographically, alone it is difficult to differentiate. No individual tooth-like structures are seen ${ }^{6}$.

Odontomas have also been classified as central or intra-osseous, which present inside the bone; peripheral or extra-osseous, which occur in the soft tissue covering the tooth-bearing portions of the jaws, and erupted odontomas according to clinical presentation ${ }^{7,8}$.

\section{Case report:}

An 8 year old child reported to the department of pedodontics with the chief complaint of partially erupted teeth in the upper front teeth region. The intra-oral examination, gave the appearance of erupting left central and lateral incisors in the space between deciduous right central incisor and deciduous left canine from the labial attached gingiva. Patient gives the history of missing deciduous left central and lateral incisor. The labial gingiva was showing mild swelling with no erythema and ulceration (Fig: 1). On thorough inspection, the erupting tooth had 2 sharp cusp like projections. On palpation, exhibited hard in consistency. Radiographic 
examination showed 3 radio-opaque bodies in between 51 and 63 (Fig: 2). The surgical removal of the 3 calcified masses were done thus, creating the path for eruption of the permanent incisors. The gross specimen consisted of three small malformed tooth like structures (Fig: 3 ). The tooth like structures were decalcified, and processed via formalin fixation. Hematoxylin and eosin (H\&E) stained sections were observed under light microscope. At magnification of X40, H\&E sections showed irregular arrangement of dentin, enamel and pulp (Fig: 4). Mature enamel is lost during decalcification procedure and hence cannot be seen in conventional hematoxylin and eosin staining. In most of the areas, dentin is showing globular arrangement. Cross section of dentinal tubules are seen throughout the dentin under X200 magnification (Fig: 5 \& 6). Scalloped outline of dentino-enamel junction was seen. Few pulpal spaces with pulpal connective tissue is observed. Enamel space is partially filled with enamel matrix in few areas. Loose myxoid connective tissue with odontogenic epithelial islands (Fig: 7) seen in close association with the lesion, and most often represents normal dental follicular tissue. Based upon the above findings, the diagnosis of compound odontome was given.

\section{Discussion:}

Odontoma is the most common type of odontogenic tumour, although some authors prefer to refer to it as hamartomas, not a true tumour ${ }^{2}$. Complex odontomas tend to occur in the posterior of the jaw and compound odontomas are more common in the anterior maxilla ${ }^{9}$. It is usually asymptomatic, but it can cause impaction and/or dental malpositioning and an increase of the volume in the area. Odontomas are usually diagnosed during the second and third decades of life ${ }^{10}$. Some sign and/ or symptom is occasionally seen - the most common condition being delayed tooth eruption.

Odontomas are typically relatively small and rarely exceed the size of a tooth in the areas where they are located. Large odontomas up to $6 \mathrm{~cm}$ or more in diameter causing expansion of the jaw are occasionally seen ${ }^{9}$.

There are two types of odontomas: complex odontomas and compound odontomas - the latter being twice as frequent as the former. Compound odontomas show a predilection for the anterior maxilla, while complex odontomas are typically found in the posterior mandibular region ${ }^{6}$.

Compound odontomas are benign lesions with a more limited growth potential than complex odontomas. Commonly, these are located between the apex of a root of a primary tooth and the crown of a permanent tooth, preventing the latter from erupting. An unerupted tooth is frequently associated with the odontoma, and the odontoma prevents its eruption. Radiologically, compound odontomes are characterized by a radiopaque mass of varying size which is composed of a number of tooth like structures in a disorderly pattern called denticles, which are miniaturized and malformed ${ }^{6}$. The complex odontoma appears as a calcified mass with the radio-opacity of tooth structure, surrounded by a narrow radiolucent rim. ${ }^{9}$

On histologic examination, Compound odontomas show tooth-like structures which resemble pulp tissue in the central portion surrounded by a dentin shell and partially covered by enamel. Complex odontomas show conglomerates without orientation of dentin, enamel, enamel matrix, cementum, and areas of pulp tissue. The capsule of connective tissue that surrounds an odontoma is similar to the follicle that covers a normal tooth. $^{1}$

The treatment of choice is surgical removal of the lesion, followed by histopathological study to confirm the diagnosis.

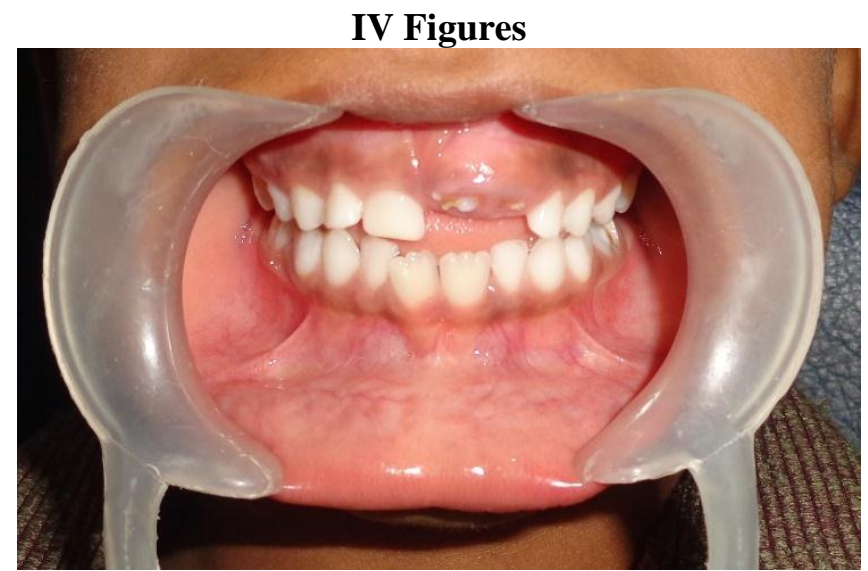

Fig: 1.Erupting compound odontoma with swelling of labial attached gingiva 


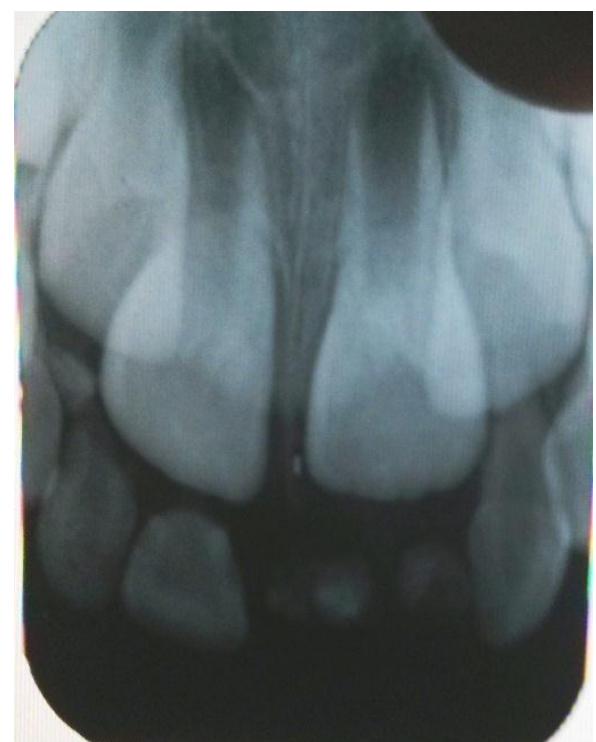

Fig: 2: Intra oral periapical radiograph showing three irregular radiopaque masses in between 51 and 63 .

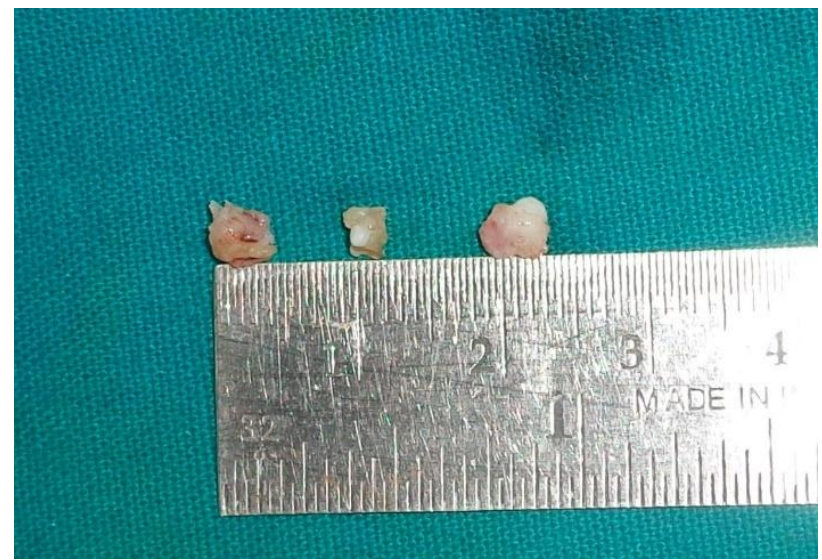

Fig: 3. Gross appearance of the specimen.

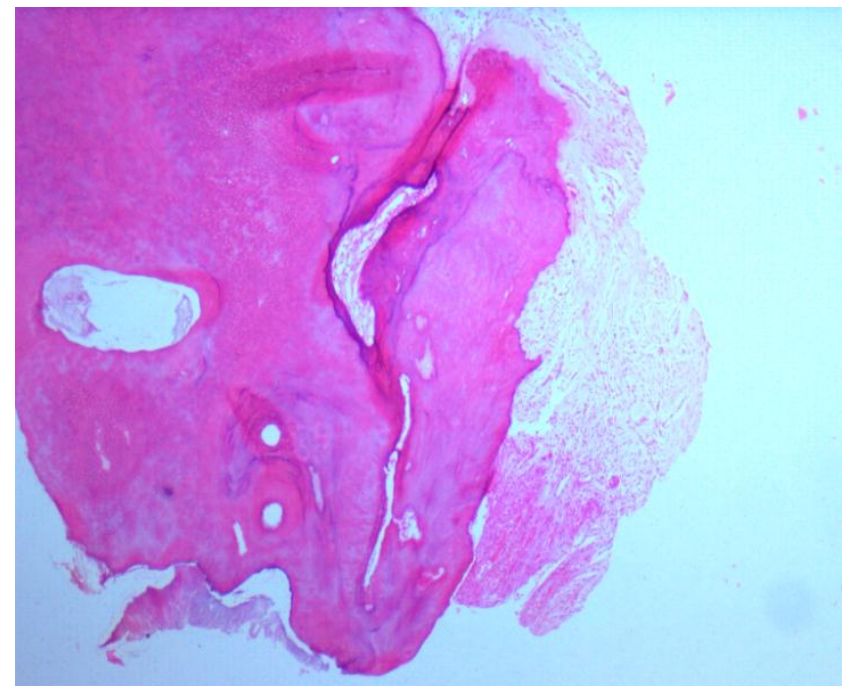

Fig: 4. Decalcified hematoxylin \& eosin stained section showing irregularly-arranged dental tissues, principally dentin and pulp. (X40 magnification) 


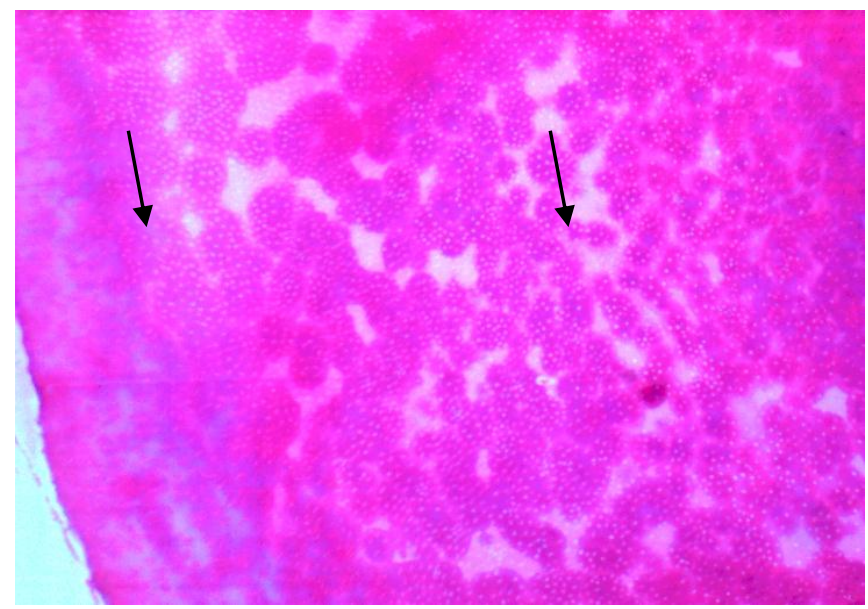

Fig: 5 Hematoxylin and eosin stained sections showing cross section of dentinal tubules (arrows). (X200 magnification)

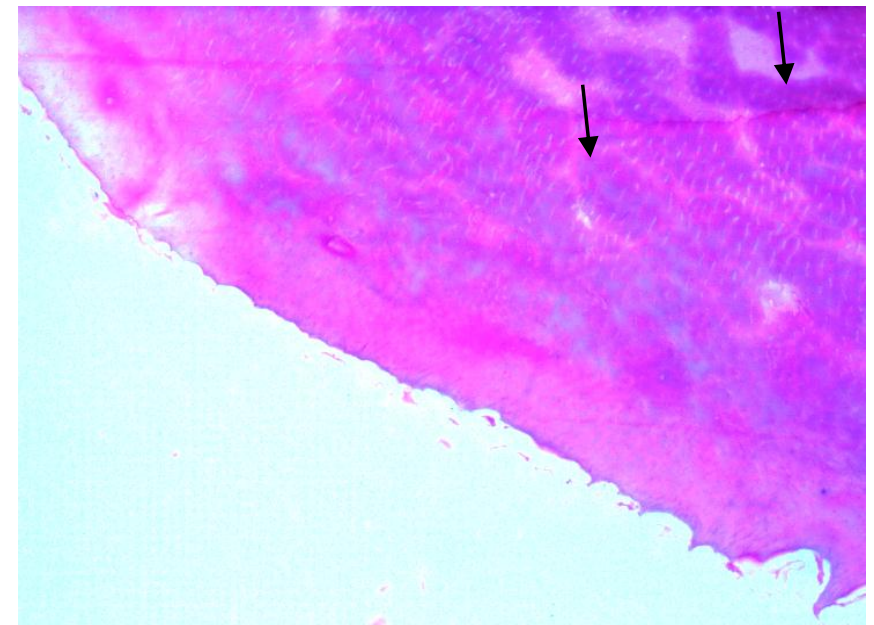

Fig: 6.Decalcified section showing scalloped outline of dentino-enamel junction and interglobular dentin(arrows) ( X 400 magnification).

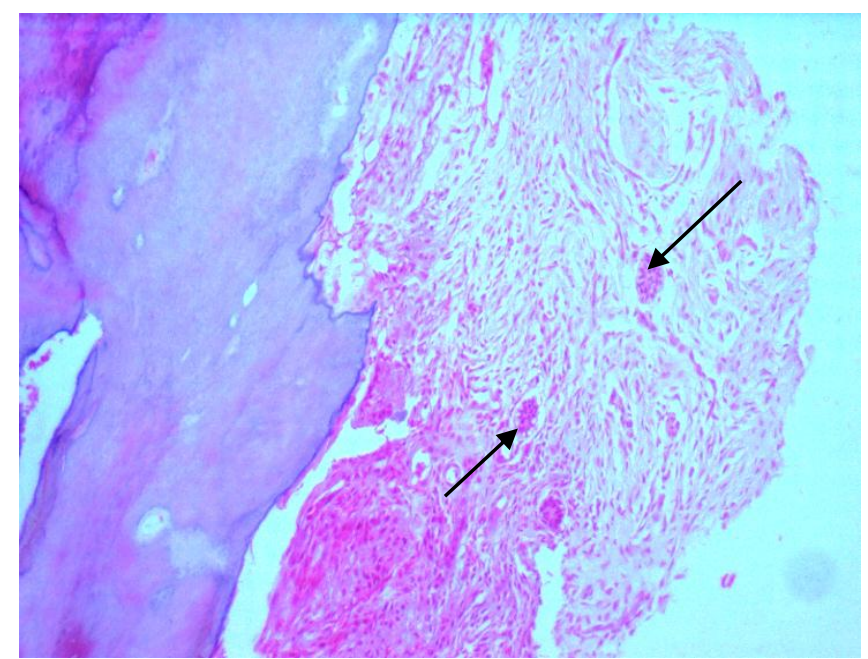

Fig: 7.Follicular tissue showing few odontogenic epithelial islands. (X200 magnification) 


\section{Conclusion:}

Most cases of compound odontomas result in the disturbance in permanent teeth eruption. This article reports a case of compound odontoma in an 8-year-old child which simulated an erupting central and lateral incisors. The prognosis of these tumours are very favorable, with a minimal tendency towards relapse. In young children, they occur in the early developing stages which may delay the eruption of permanent teeth. So, early diagnosis and treatment of compound odontoma is necessary in order to prevent any malocclusion so that permanent teeth may erupt in normal pattern.

\section{References}

[1] Shafer WG, Hine MK, Levy BM. A Textbook of Oral Pathology 6th ed. Philadelphia: Elsevier Noida; 2009. pp. 287-90

[2] Cohen DM, Bhattacharyya I. Ameloblastic fibroma, ameloblastic fibro-odontoma, and odontoma. Oral Maxillofac Surg Clin North Am. 2004;16:375-84.

[3] Shekar S E, Rao RS, Gunasheela B, Supriya N. Erupted compound odontome. J Oral MaxillofacPathol 2009;13:47-50

[4] Preetha A, Balikai BS, Sujatha D, Pai A, Ganapathy KS. Complex odontoma. Gen Dent.2010;58:e100-2.

[5] Serra-Serra G, Berini-Aytés L, Gay-Escoda C. Erupted odontomas: A report of three cases and review of the literature. Med Oral Patol Oral Cir Bucal. 2009 Jun 1;14 (6)::E299-303.

[6] Philipsen H, Reichart P, Praetorius F. Mixed odontogenic tumours and odontomas. Considerations on interrelationship. Review of the literature and presentation of 134 new cases of odontomas. Oral Oncol. 1997;32:86-99.

[7] Junquera L, de Vicente JC, Roig P, Olay S, Rodríguez-RecioIntraosseus odontoma erupted into the oral cavity: An unusual pathology. Med Oral Patol Oral Cir Bucal 2005; 10:248-51.

[8] Singh V, Dhasmana S, Mohammad S, Singh N. The odontomes: Report of five cases. Natl J MaxillofacSurg 2010; 1:157-60.

[9] Neville, Damm, Allen, Bouquot. Oral and Maxillofacial Pathology $2^{\text {nd }}$ ed. 2004; pg.631-32.

[10] Kaneko M, Fukuda M, Sano T, Ohnishi T, Hosokawa Y. Microradiographic and microscopic investigation of a rare case of complex odontoma. Oral Surg Oral Med Oral Pathol Oral Radiol Endod 1998;85:131-4. 